\title{
Development and integration of adaptive underfrequency load shedding into the smart grid
}

\author{
R. Petrichenko, V. Chuvychin, A. Sauhats, V. Strelkovs \\ Faculty of Power and Electrical Engineering \\ Riga Technical University \\ Riga, Latvia \\ chuvychin@eef.rtu.lv, sauhatas@eef.rtu.lv
}

\begin{abstract}
The objective of underfrequency load shedding (UFLS) is to balance generation and load in case of a significant drop in frequency. Conventional UFLS schemes rely on parameters that are obtained by analyzing predefined scenarios that might not coincide with the actual emergency process. Improved UFLS scheme is presented in the paper that estimates accurate values of active power deficiency thus presenting an opportunity to enhance the efficiency and reliability of power system operation. The theory of the presented scheme is verified by computer modelling. Integration of the new UFLS scheme into the smart grid where it can be most effective by taking advantage of digital communication systems and smart meters is also discussed.
\end{abstract}

Keywords—automatic frequency control, smart grids.

\section{INTRODUCTION}

Underfrequency load shedding (UFLS) is the last automated measure associated with a decline of frequency. It is designed to rapidly balance the demand of generation and load as large frequency deviations degrade load performance, overload transmission lines and can even lead to system collapse.

Many different types of UFLS schemes are presented in literature and used by electric utilities. Typically, these schemes are classified as static and dynamic UFLS. Static load shedding disconnects constant amount of load at every stage, whereas dynamic load shedding disconnects varying amount of load, at each step taking into consideration frequency parameters of the system and the magnitude of disturbance. Even though the dynamic under-frequency load shedding schemes are more flexible, most real-world UFLS schemes are static [1].

Unfortunately, static UFLS often sheds too much load or insufficient amount of load due to a combination of the following reasons:

- Parameters of load-shedding scheme are established by studying possible system scenarios that may require load shedding [2], [3]. The manner in which most systems can become generation deficient or separated are numerous. As a result, the effectiveness of load shedding system can vary considerably depending on

This research has been partly supported by the State Research Program "LATENERGI". the character of disturbance.

- Volume of load that has to be allocated to underfrequency control is estimated differently. Emergencies that result in operation of under-frequency relays do not occur on often therefore acquiring of experience is relatively slow [3]

- Previously, the reference load for load shedding was determined according to the typical load situations during particular seasons, for example. Due to the increasing impact of distributed generation, this approach is no longer suitable for the reference load estimation [4].

- Underfrequency load shedding plans are not coordinated among transmission system operators (TSOs). The settings of load shedding are chosen differently by different TSOs according to the corresponding documents: laws, grid codes, internal rules.

- The load-shedding relays mostly are installed at distribution or subtransmission level. Those feeders, that meet the necessary power that is needed for loadshedding automation, are selected and assigned to appropriate steps of the load-shedding relays [2]. Actual feeder loading is rarely controlled in static UFLS schemes and consequently the size of disconnected load can vary.

- To enhance the operation of under-frequency loadshedding systems, rate-of-change of frequency (ROCOF) relays are sometimes applied. The rate-ofchange of frequency clearly reflects power imbalance. The use of ROCOF relays provides a more robust load shedding system. It allows shedding load in a timelier manner based on the severity of the active power imbalance condition. The oscillating nature of frequency decay rates, however, can cause incorrect operation of ROCOF relays. Moreover, the settings for ROCOF relays are rather difficult to estimate [3].

Many different developments are encountered in scientific publications regarding underfrequency load shedding. They range from utilization of communication technologies (SCADA, WAMS) to application of fuzzy logic, neural 
networks, game theory, and genetic algorithms. Challenges posed by increasing proportion of distributed and renewable power sources are also dealt with [3-5].

In this publication authors turn their attention to the direct calculation of active power deficit as well as of active power excess. Knowledge of accurate amount of disturbance can greatly simplify the decision-making and enhance the efficiency and reliability of power system operation.

Common approach to estimate active power deficiency is to utilize power balance equation (1) characterizes frequency deviation in case of active power imbalance. After an event, an optimal load shedding system should trip enough load so that the $\Delta \mathrm{P}$ term is equal to zero.

$$
\frac{T_{J}}{\omega_{R}} \cdot \frac{d \Delta \omega}{d t}=P_{M}(\omega)-P_{E}(\omega)=\Delta P,
$$

where $\mathrm{P}_{\mathrm{M}}$ is mechanical turbine power in pu, $\mathrm{P}_{\mathrm{E}}$ is electrical power in pu, $\Delta \mathrm{P}$ is active power imbalance in $\mathrm{pu}, \mathrm{T}_{\mathrm{J}}$ is inertia constant in sec, $\omega_{R}$ is rated rotational speed in $\mathrm{rad} / \mathrm{sec}, \Delta \omega$ is deviation from the rated rotational speed in $\mathrm{rad} / \mathrm{sec}$.

The rate of frequency decline at the beginning of the disturbance (initial slope of frequency decline) can accurately reflect the magnitude of the disturbance (2). This approach has already been considered in many publications. Two of them that are often referenced and represent different time periods are [6] and [7].

$$
\left.\frac{d \Delta \omega}{d t}\right|_{t=0}=\frac{\Delta P \cdot \omega_{R}}{T_{J}}
$$

Application of initial slope of frequency decline has several shortcomings:

- The volume of active power deficit according to (2) is accurately characterized only at the first instant of frequency deviation if the inertia constant is precisely known. In the subsequent time moments a wide range of parameters influence frequency and estimation of deficit with (2) becomes increasingly inaccurate. As UFLS typically sheds load only after frequency declines below some predefined value that is estimated from system studies. Load disconnection usually has time delay that ranges from milliseconds to tens of seconds. Moreover, extra time delays appear do to internal operational time of protective automation devices as well as of circuit breakers. It means that the actual disconnection takes place too late for equation (2) to be applicable.

- In emergency situation frequency can briefly steady itself at non-rated value thus precluding any of the deficit estimation methodologies that rely solely on rate-of-change parameter.
- Methodologies that relay on (2) type of equations are not reliable also in case of relatively slow deviations of frequency.

- Different load disconnection options are available - for instance, all of the load can be disconnected at once; most of the load can be disconnected at first and then the remaining load can be used to fine-tune the frequency; entire load can be split into equal parts and gradually disconnected with the same time delays, etc. It is clear that equation (2) is not reliable if the whole load is not disconnected instantly.

- From the literature review, it can be found that the methodologies of deficit estimation that are based on the first deviation instant are researched in just a few particular scenarios, most typical of which are isolated systems. It must be emphasized that different systems react differently on frequency deviation and it is possible than in case of isolated system with some CHP plants the method operates well but in other systems it cannot not cope, for instance, with large penetration level of renewable resources.

In the second chapter of this paper authors present a UFLS scheme that accomplishes several significant tasks - it estimates deficit (and excess) values throughout the transient process allowing splitting the load disconnection action into several steps for safer more universal control action. Moreover, the offered UFLS automatically adapts to the actual situation in power system mainly by correcting the amount of load that should be disconnected. As it was mention before (2) based methodologies are not capable of dealing with steady state offnominal frequencies or with frequencies that vary slowly, therefore the offered new UFLS works fully-fledged in these situations as well.

In third chapter authors analyze the application of the suggested UFLS.

In the fourth chapter authors deal with the integration of the new UFLS scheme into the smart grid, taking into account that the smart grid is an environment in which the considered UFLS can be most effective by utilizing two-way communication between the utility and its customers.

\section{DEVELOPMENT OF ADAPTIVE UNDERFREQUENCY LOAD SHEDDING SCHEME}

The sequence of events how active power deficit or excess is determined is showed below.

A) Mathematical description for $\mathrm{P}_{M}(\omega)$ component of (1) is obtained.

See, for example Fig. 1, where $P_{M}\left(\omega_{R}\right)$ is active power setpoint in $\mathrm{pu}, \mathrm{F}_{\mathrm{H}}$ is a fraction of total power generated by turbine, $\mathrm{T}_{\mathrm{R}}$ is reheat time constant in sec, and $\mathrm{K}_{\mathrm{M}}$ is mechanical power gain factor.

A low-order frequency response model of reheat steam turbine unit is chosen as an example here because it is well known and is often featured in publications and textbooks. The 
methodology presented in this chapter is applicable to any necessary frequency response model.

From Fig. 1 we get:

$$
P_{M}(\omega)=\left(P_{M}\left(\omega_{R}\right)-\frac{\Delta \omega}{R}\right) \cdot \frac{T_{R} \cdot F_{H} \cdot s+1}{T_{R} \cdot s+1} \cdot k_{M}
$$

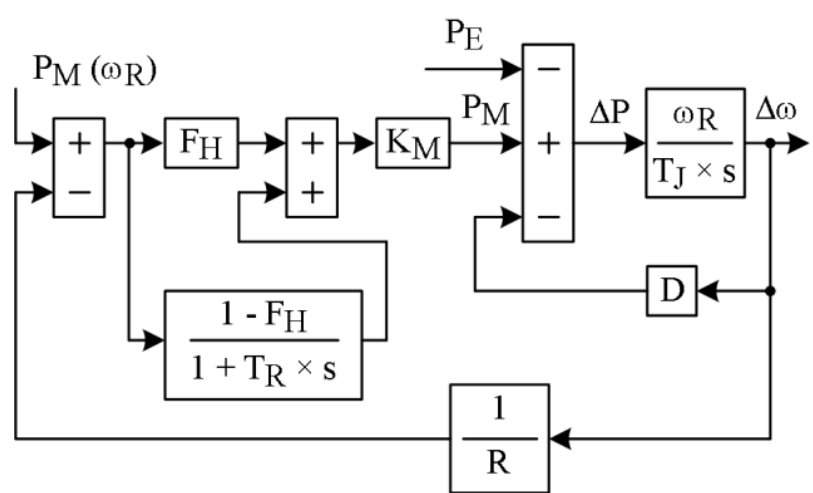

Fig. 1. A low-order frequency response model of reheat steam turbine unit

B) The necessary input parameters are determined. There are parameters that are known, such as rated rotational frequency $\left(\omega_{\mathrm{R}}\right)$. There are parameters that can be obtained from measurements, such as frequency $(\omega, \mathrm{f})$. Some parameters can be easily calculated, such as rate-of-change of rotational frequency $(\mathrm{d} \Delta \omega / \mathrm{dt})$. A number of parameters are given in passport data $\left(\mathrm{T}_{\mathrm{J}}\right)$ etc.

C) Electrical power $P_{E}(\omega)$, rotational speed $\omega$, its deviation $\Delta \omega$ and derivative $\mathrm{d} \Delta \omega / \mathrm{dt}$ are continuously measured at the generator bus.

D) Expression for $P_{M}(\omega)$ is inserted into (1). Here for convenience derivative is replaced with Laplace operator "s":

$$
\begin{aligned}
& \frac{T_{J}}{\omega_{R}} \cdot s \cdot \Delta \omega= \\
& =\left(P_{M}\left(\omega_{R}\right)-\frac{\Delta \omega}{R}\right) \cdot \frac{1+F_{H} \cdot T_{R} \cdot s}{1+T_{R} \cdot s} \cdot k_{M}-P_{E}(\omega)
\end{aligned}
$$

E) $P_{M}\left(\omega_{R}\right)$ is estimated from (4) as the only unknown quantity.

F) Electrical power at rated rotational speed $P_{E}\left(\omega_{R}\right)$ is calculated using the measurements of $\omega$ and $P_{E}(\omega)(5)$ :

$$
P_{E}\left(\omega_{R}\right)=P_{E}(\omega)-D \cdot \Delta \omega
$$

G) Deficit or excess is continuously estimated.

$$
D E F=P_{E}\left(\omega_{R}\right)-P_{M}\left(\omega_{R}\right)
$$

H) If there are several power plants (if this methodology is not used in isolated system) then the previous steps are repeated for each power plant.

I) When the deficit is known it can be easily transformed into corresponding control activity. If deficit is calculated at several power plants, the control activity can be coordinated by centralized automatic operator.

\section{ANALYSIS OF SUGGESTED UFLS SCHEME}

The proposed new method for active power deficiency estimation requires knowledge of the frequency response model for every considered generating unit as well as the capability to solve system of differential equations.

Authors have tested the suggested method for a number of specific issues that are listed below.

1) It is inevitable that at least some of the data that is necessary for deficit estimation is not exactly known.

Let us take as an example a single reheat steam turbine unit (see Fig. 1) with the following parameters: $T_{J}=8 \mathrm{~s}, \mathrm{D}=1, \mathrm{R}=$ $0.05, \mathrm{~K}_{\mathrm{M}}=0.95, \mathrm{~F}_{\mathrm{H}}=0.3, \mathrm{~T}_{\mathrm{R}}=8 \mathrm{~s}$. As we are interested only in a change in $\mathrm{P}_{\mathrm{E}}$, we can assume initially $\mathrm{P}_{\mathrm{M}}=0$ pu and $\mathrm{P}_{\mathrm{E}}=0$ pu. In this one machine system, let us simulate a sudden increase of $\mathrm{P}_{\mathrm{E}}$ by $0.1 \mathrm{pu}$ and calculate active power deficiency for three different values of $T_{J}$. The resulting frequency transient is shown in Fig. 2.

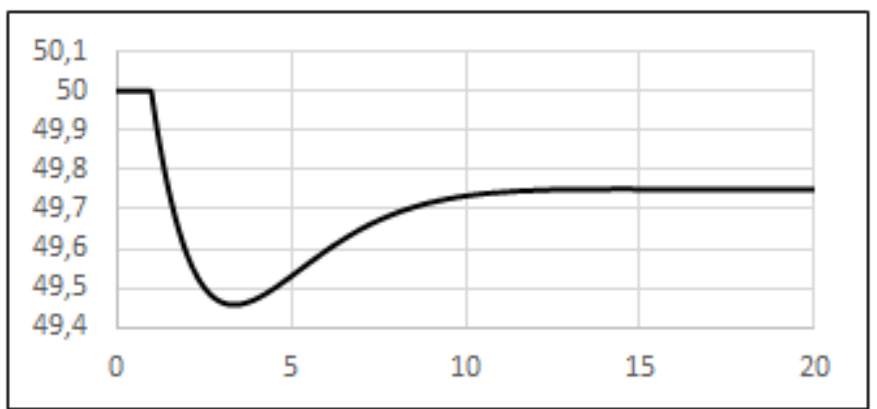

Fig. 2. Frequnecy transient due to $0.1 \mathrm{pu}$ active power disturbance (frequency vs time)

For $\mathrm{T}_{\mathrm{J}}=8 \mathrm{~s}$ deficiency is estimated correctly. With incorrect inertia constant ( $7 \mathrm{~s}$ or $9 \mathrm{~s})$, however, calculation errors appear instantly (Fig. 3) as it is expected from (2).

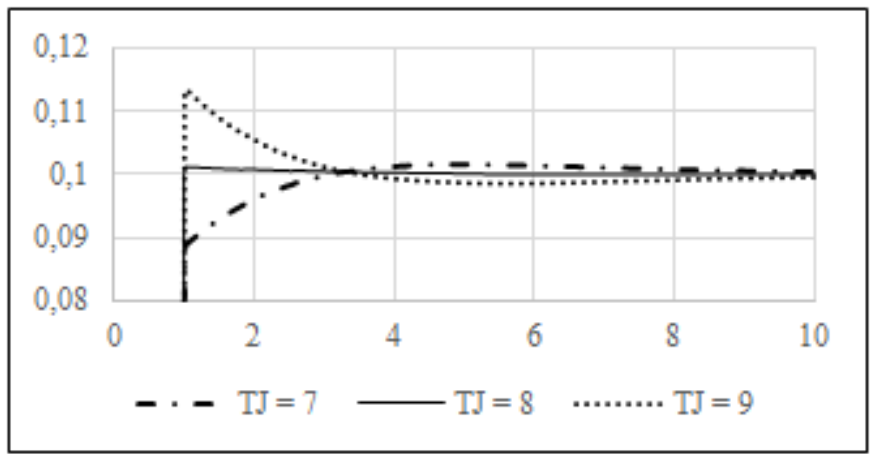

Fig. 3. Influence of $\mathrm{T}_{\mathrm{J}}$ on active power deficiency estimation (calculated deficiency vs time) 
After the disturbance, turbine speed controller increases active power output and frequency starts to stabilize. Frequency rate of change decreases and calculation errors diminish because $T_{J}$ is a coefficient at frequency derivative.

Calculation errors appear instantly also in case of wrongly assumed D (Fig. 4):

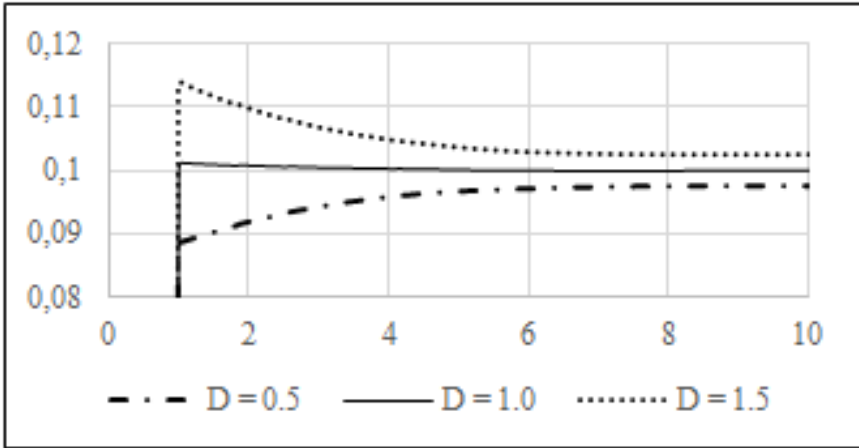

Fig. 4. Influence of D on active power deficiency estimation (calculated deficiency vs time)

Other parameters (for particular frequency response model) exert less influence on deficiency estimation as the errors express themselves gradually in time (Fig. 5).

With fast control, action errors do not have enough time to reach unacceptable levels. If the load is shed in several steps then the errors are dispersed and decrease with each loadshedding step.

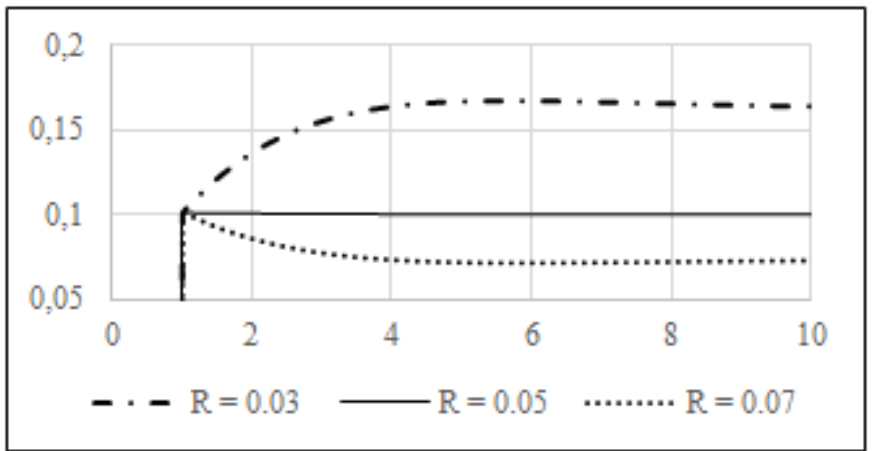

Fig. 5. Influence of $\mathrm{R}$ on active power deficiency estimation (calculated deficiency vs time)

2) In actual power system there are many power plants. It means that the deficit can be calculated in any of them, but not necessarily at all of them.

To show how it affects the offered methodology, we will look at the simple system (Fig. 6.) with three equal power plants (with the same parameters as in previous case) connected in series through identical transmission lines:

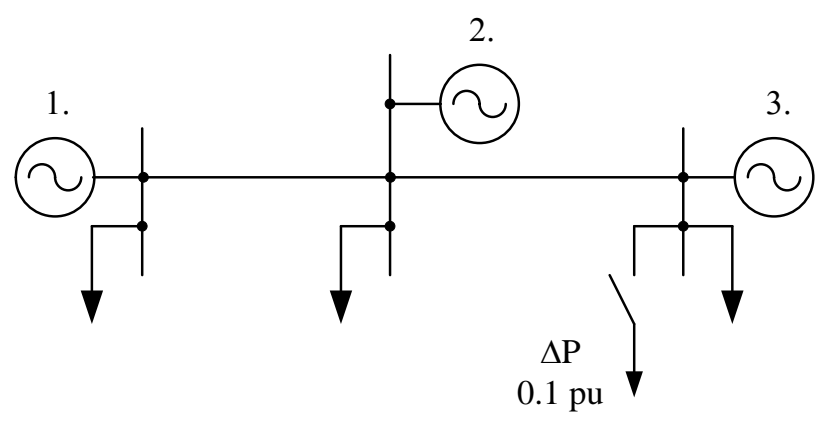

Fig. 6. Active power deficiency in a three machine system

Simulation results of a sudden increase of PE by 0.1 pu on a bus of a third power plant are shown in Fig 7, where DEF1, DEF2, DEF3 is continuously estimated deficiency at the corresponding bus and " $1+2+3$ " is the sum of all estimated deficiencies. Load is not disconnected during the simulation.

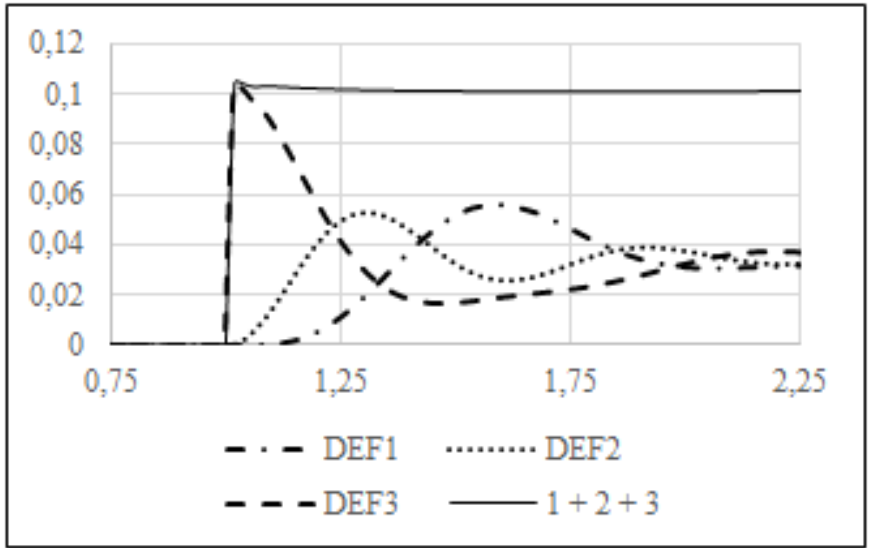

Fig. 7. Active power deficiency in a three machine system (calculated deficiency vs time)

The disturbance gradually spreads through the whole system. It can be seen that the total level of active power deficiency at any moment of time is equal to the applied disturbance. Each station notices just some fraction of total deficit (according to its electrical distance from the disturbance and frequency response).

Although the turbine speed controllers increase generated power and frequency stabilizes (in a similar manner as in Fig. 2 ), total deficiency stays the same during the whole process, because in order to restore the rated frequency either load has to be shed by $0.1 \mathrm{pu}$, or generator set-points should be increased in total by $0.1 \mathrm{pu}$.

There are other significant points that should be analyzed but they too extensive and will be elaborated in further publications. For example the analysis of the best way to shed load - at once, gradually, locally, globally etc. This requires extensive system studies and the result is highly depended on the particular system.

As this methodology allows to calculate the active power excess as well (in the same way as deficit), the control action can involve starting of generators that can also be carried out very differently. 
There are always some practical limitation as well than can restrict the methodology, such as overloading of lines, cables, or other equipment. With every control action, (disconnection of load or start of generator) power flows are redistributed in power system.

Eventually, although the frequency is global parameter and is mainly dependent on active power balance, every control action influences voltage as well.

\section{INTEGRATION OF DEVELOPED UNDERFREQUENCY LOAD SHEDDING SCHEME INTO THE SMART GRID}

In recent years the term Smart Grid is increasingly used in different engineering fields. There are many papers describing the application of Smart Grid devices in electrical generation, transmission, and distribution, as well as in communication technologies. Many papers describe the possibility of centralized control systems [8] that integrate Smart Grid based technology, known as Smart Metering system [9]. This system utilizes electronic devices that record consumption of electric energy, allow certain control of load and enable two-way communication between the meter and centralized control system. Application of such interactive technology enhances the control of power system in normal and emergency operating modes.

In this chapter, we will consider a power system that is split into several districts (Fig. 8) where each district is equipped with an interactive measuring device that is connected to the control center - "Operator". Deficiency detection and according actions from "Operator" are main functions of each power districts' measuring device.

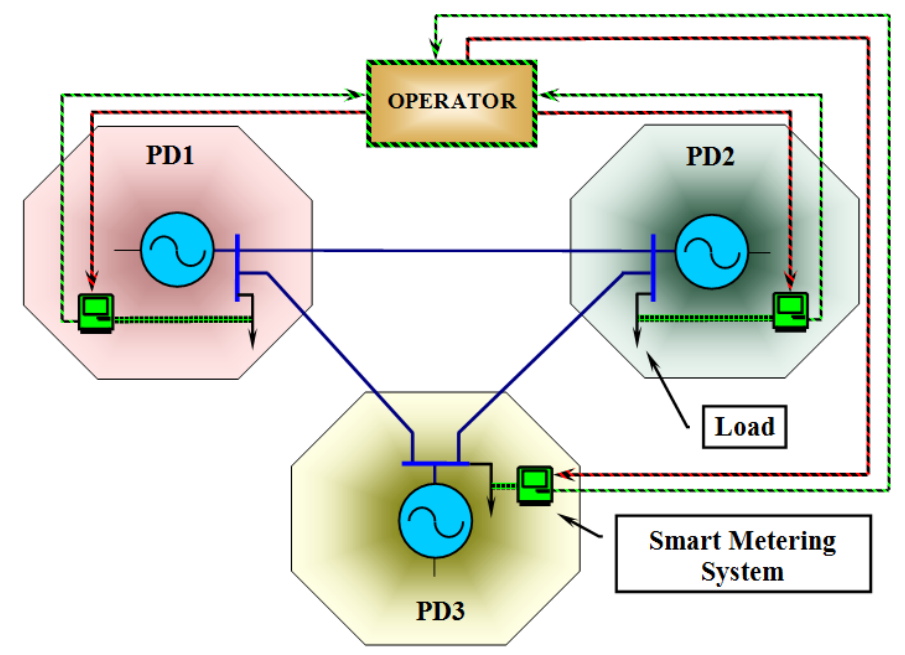

Fig. 8. Suggested control system diagram of three power districts

The suggested protection scheme (let us call it Smart Under-frequency Load Shedding - SUFLS) consists of the following operational cycles: determination of active power deficiency value, storage of the information about deficiency value and its location, estimation of number of power districts where control action should be applied, and calculation of optimal scenario for disconnection of load and disconnection of load feeders.

\section{A. Detection of active power deviance}

Active power deficiency is determined by each smart metering system according to the methodology that is presented in chapter 2 .

\section{B. Registration of deficiency and its location}

When the deficiency is determined, its value along with its location is sent to the "Operator" where this information is further used to compute the necessary control action.

\section{Calculation of number of power districts for deficiency compensation}

System detects and record deficiency, its value and district. Estimation of power districts quantity required for the deficiency compensation is a next step of developed UFLS. Fig. 9 displays flowchart of suggested UFLS calculation procedure - algorithm.

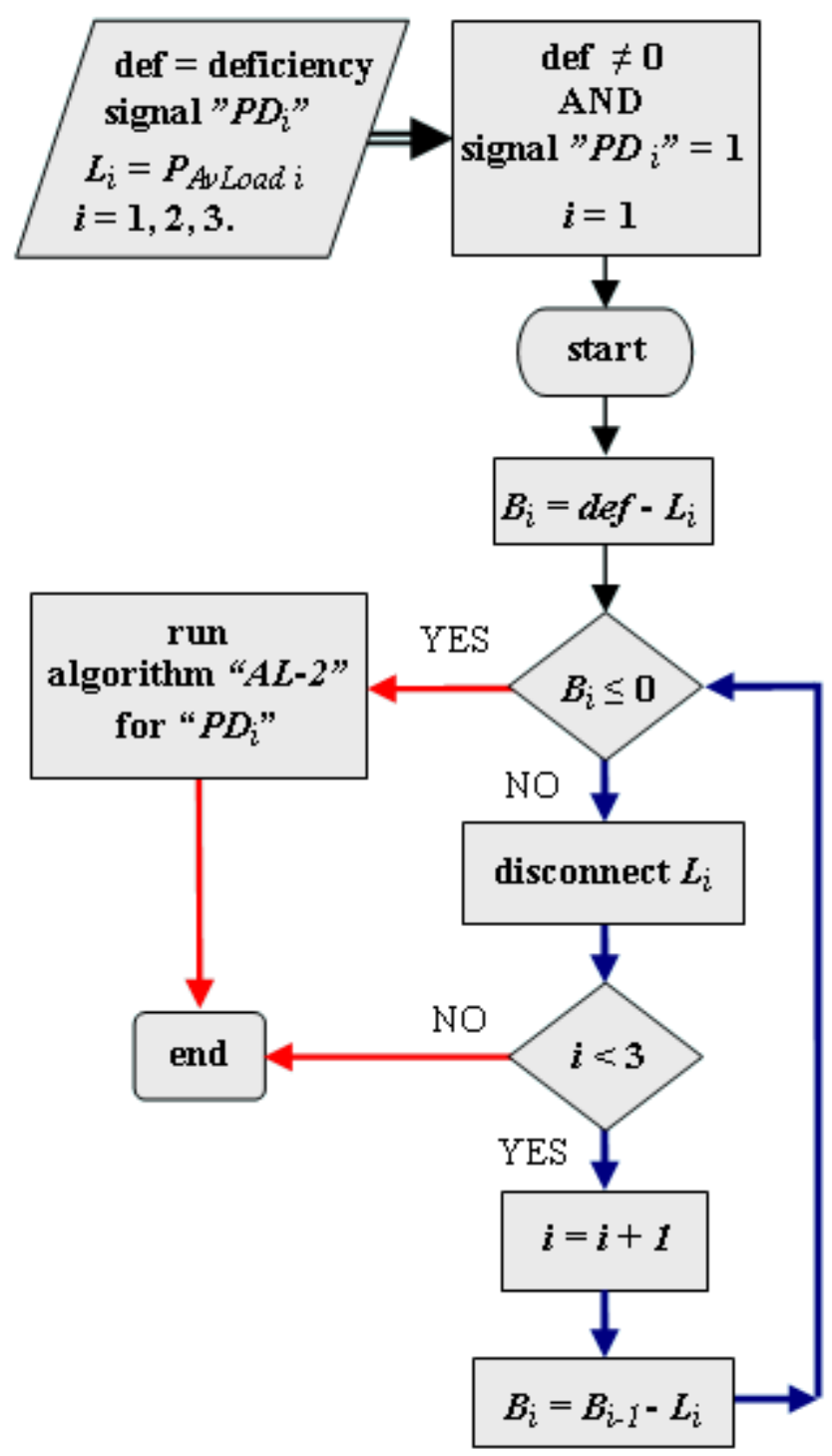

Fig. 9. Algorithm "AL-1" flowchart 
Each power district, by interactive metering system, permanently sends information about produced and consumed active power. Information threads continuously processed by power system control center 'Operator'.

Opportunity of deficiency compensation by load shedding $\left(\mathrm{P}_{\text {AvLoad-i }}\right)$ procedure is a main goal of smart UFLS (SUFLS) automation. Positive or neutral difference between detected deficiency and available load value, runs algorithm 'Al-2' (see Fig. 9), which calculates optimal load shedding procedure.

Otherwise, current power district's available load are shaded and estimation of remaining deficiency value $\mathrm{B}_{1}$ takes place. Algorithm 'AL-1' goes to next power district and there the probability calculation of deficiency compensation is calculated.

Deficiency compensation procedure is performed as long as active power disbalance is not fully eliminated.

\section{Calculation of optimal scenario for load disconnection}

Aforementioned theoretical description should be proofed by case study. For example, let us consider interactive power system with three power district, which are equipped by 10 load feeders each [10]. Operation comparison in emergency of two developed models (existing UFLS and developed SUFLS in Matlab/Simulink software) are presented in figure 10.

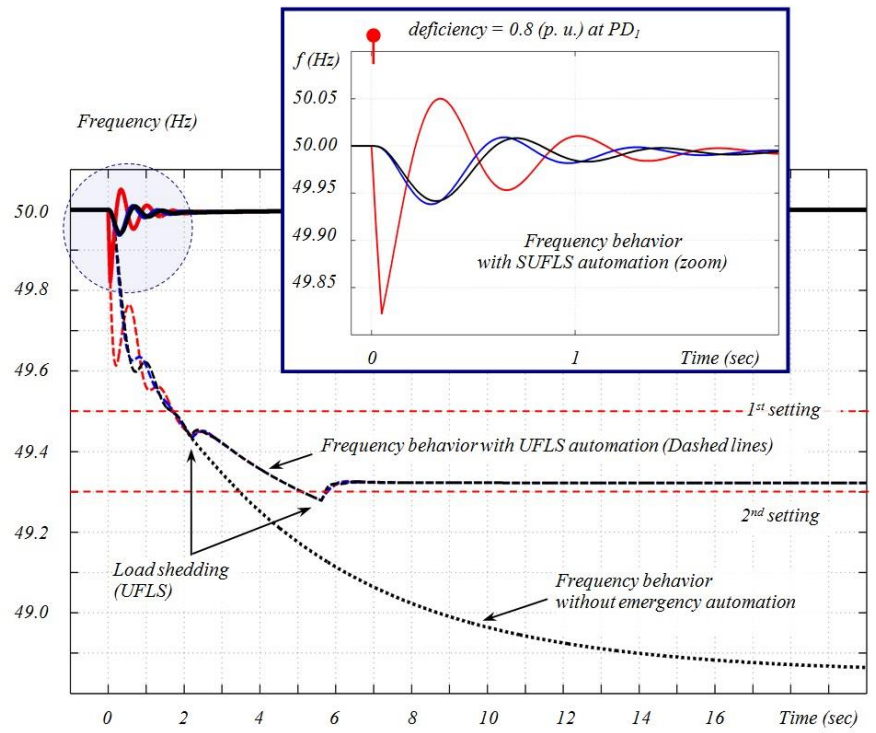

Fig. 10. Frequency behavior for different types of load shedding automation (1- Frequency behavior with conventional UFLS automation, 2 - Frequency behavior without emergency automation, zoomed region - SUFLS).

Effectiveness of the proposed SUFLS automation is obvious. Developed automation sheds load in districts accordingly deficiency location.

\section{CONCLUSIONS}

A new UFLS methodology that allows exact estimation of active power deficit or excess is presented. Important quality of the suggested scheme is its capability to adapt itself to changing conditions in power system. Although it is relatively complicated, requires many input parameters and solution of differential equations it can be realized, using modern microprocessor-based numerical devices.

The developed scheme offers an opportunity to enhance UFLS principles. It does not require waiting until frequency drops below preset frequency level. For example, since the frequency during the whole system disturbance on November 4, 2006 in Southeastern area was above the first threshold of load shedding the defense plans were not activated. The new scheme also allows restoring nominal value of frequency and it presents valuable information about the magnitude of the disturbance to TSO's.

Application of smart metering systems with two-way communication between the utility and its customers can enhance the control of power system in normal and emergency operating modes.

\section{REFERENCES}

[1] Hassan Bevrani, Robust power system frequency control, Springer, 2009, pp. 175-176.

[2] Stanley H. Horowitz, Arun G. Phadke, Power systems relaying, 3rd ed., John Wiley \& Sons, 2008, pp. 267-268.

[3] J. Lewis Blackburn, Thomas J. Domin, Protective relaying - principles and applications, 3rd ed., CRC Press, 2006, pp. 531-533.

[4] ENTSO-E. "Technical background for the low frequency demand disconnection (LFDD) requirements," November 2014, p. 5.

[5] Aboli Kulkarni, James Payne, Peter Mistretta, "SCADA based under frequency load shedding integrated with rate of frequency decline," IEEE Transactions on Industry Applications, Volume 51, Issue 2, pp. 1360 - 1368, 2015.

[6] P. M. Anderson, M. Mirheydar, "An adaptive method for setting underfrequency load shedding relays," IEEE Transactions on Power Systems, Volume 7, Issue 2, pp. 647 - 655, May 1992.

[7] Vladimir V. Terzija, "Adaptive underfrequency load shedding based on the magnitude of the disturbance estimation," IEEE Transactions on Power Systems, Volume 21, Issue 3, August 2006.

[8] U. Rudez, R. Mihalic, "Analysis of Underfrequency Load Shedding Using a Frequency Gradient," IEEE Trans. Power Syst., Vol. 26, No. 2, pp 565 - 575, Apr., 2011.

[9] P. H. Nguyen, W. L. Kling, J. M. A. Myrzik, "Power flow management in active network," IEEE Bucharest Power Tech Conference, Bucharest, Romania, 29 June - 2 July, 2009.

[10] R. Petrichenko, V. Chuvychin, A. Sauhats, "Coexistence of Different Load Shedding Algorithms in Interconnected Power System," 12th International Conference on Environment and Electrical Engineering (EEEIC), Wroclaw, Poland, 5-8 May 2013.

This is a post-print of a paper published in Proceedings of the 2017 IEEE International Conference on Environment and Electrical Engineering and 2017 IEEE Industrial and Commercial Power Systems Europe (EEEIC / I\&CPS Europe), 